\title{
WSI-Mindestlohnbericht 2012 - Schwache Mindestlohnentwicklung unter staatlicher Austeritätspolitik
}

\author{
Die Entwicklung der Mindestlöhne hat sich im Jahr 2011 gegenüber den Vorjahren noch \\ einmal deutlich verlangsamt - meist wurden die Mindestlöhne nur geringfügig ange- \\ hoben oder sogar gänzlich eingefroren. In den meisten europäischen Ländern erlitten die \\ Mindestlohnbezieher zum Teil deutliche Reallohnverluste. Die Mindestlohnpolitik \\ wurde im Rahmen des aktuellen Krisenmanagements in der Europäischen Union zum \\ Bestandteil einer allgemeinen Austeritätspolitik. Angesichts ihrer Signalwirkung für \\ die allgemeine Lohnentwicklung werden auf diese Weise die ökonomischen Stagnations- \\ tendenzen in Europa weiter verschärft.
}

THORSTEN SCHULTEN

\section{Einleitung}

Mit den Beschlüssen des CDU-Parteitages im November 2011 ist die Mindestlohndebatte in Deutschland in eine neue Phase getreten. ${ }^{\bullet}$ Fortan geht es nicht mehr um die Frage, $o b$ in Deutschland ein gesetzlicher Mindestlohn eingeführt werden soll, sondern wie die Festlegung, Ausgestaltung und Reichweite dieses Mindestlohns aussehen soll. Umso wichtiger ist es, die konkreten Erfahrungen anderer Länder aufzuarbeiten und für die deutsche Debatte nutzbar zu machen.

Gesetzliche Mindestlöhne gehören bereits seit Langem in vielen Ländern zu den etablierten Instrumenten bei der Regulierung des Arbeitsmarktes. Nach Angaben der Internationalen Arbeitsorganisation (ILO) verfügen weltweit mehr als 100 Staaten über gesetzliche Mindestlohnregelungen (ILO 2010). Das Wirtschafts- und Sozialwissenschaftliche Institut (WSI) berichtet seit einigen Jahren im Rahmen seines jährlich erscheinenden WSI-Mindestlohnberichts über die aktuelle Entwicklung von Mindestlöhnen im europäischen und außereuropäischen Ausland. Die Grundlage dieser Berichte bildet die WSI-Mindestlohndatenbank, in der mittlerweile Daten für 30 Länder enthalten sind. ${ }^{2}$ Hierzu gehören alle 20 von 27 EU-Staaten, die über einen allgemeinen gesetzlichen Mindestlohn verfügen, die EUBeitrittskandidaten Kroatien und Türkei sowie acht weitere außereuropäische Länder (Argentinien, Australien, Brasilien, Japan, Kanada, Korea, Neuseeland und die USA).

\section{Gesetzliche Mindestlöhne zum}

\section{Januar 2012}

Bei der Höhe gesetzlicher Mindestlöhne zeigen sich sowohl innerhalb als auch außerhalb Europas erhebliche Unterschiede. Gemessen in Euro lassen sich im Hinblick auf die Mindestlohnniveaus innerhalb der EU drei Gruppen identifizieren (Abbildung 1): Die erste Gruppe mit relativ hohen Mindestlöhnen umfasst insgesamt sechs Staaten aus Westeuropa. Das höchste Mindestlohnniveau mit einem Wert von 10,41 € pro Stunde findet sich in Luxemburg, das seit Anfang 2011 als erstes europäisches Land die 10-€-Marke überschritten hat. Der zweithöchste Mindestlohn existiert mit 9,22 € pro Stunde in Frankreich, gefolgt von den Niederlanden mit $8,88 €$, Belgien mit $8,75 €$ und Irland mit $8,65 €$. Mit einem Mindestlohn von 7,01€ pro Stunde bildet Großbritannien das Schlusslicht der europäischen Spitzengruppe. Allerdings wird der in Euro gemessene Betrag des britischen Mindestlohns stark durch den Wechselkurs des

Vgl. hierzu den Beitrag von Reinhard Bispinck in diesem Heft.

2 Eine aktuelle elektronische Version der WSI-Mindestlohndatenbank findet sich unter: http://www. boeckler.de /pdf/ta_mindestlohndatenbank.pdf. Zu den Unterschieden und Abgrenzungen der bestehenden Mindestlohndatenbanken von EUROSTAT, ILO, OECD und WSI vgl. Schulten 2009. 
britischen Pfundes verzerrt, das seit 2007 gegenüber dem Euro um fast $30 \%$ abgewertet wurde. Ohne diese Abwertung würde der Mindestlohn in Großbritannien heute bei $8,88 €$ pro Stunde liegen und hätte damit einen mit den anderen westeuropäischen Ländern vergleichbaren Wert.

In einer zweiten Gruppe mit Mindestlöhnen zwischen 2 und $6 €$ pro Stunde befinden sich insgesamt sechs EUStaaten, vorwiegend aus Südeuropa. Der Spitzenreiter dieser mittleren Gruppe ist Slowenien mit einem Mindestlohn von $4,41 €$ pro Stunde, gefolgt von Griechenland mit 4,34€, Malta mit 3,96€ und Spanien mit 3,89€. Am Ende dieser Gruppe liegen Portugal mit 2,92 € und Polen mit 2,10€, das zum 1. Januar 2012 erstmals die 2-€-Marke überschritten hat. Die dritte Gruppe mit Mindestlöhnen unterhalb von $2 €$ umfasst ausschließlich Länder aus Mittel- und Osteuropa. Das Mindestlohnniveau bewegt sich hier in der Mehrzahl der Länder zwischen 1,50€ und 2,00€ pro Stunde. Lediglich in Bulgarien und Rumänien liegt der Mindestlohn nach wie vor unterhalb von $1,00 €$ pro Stunde.

Außerhalb der EU hat von den hier berücksichtigten Ländern Australien den höchsten Mindestlohn, der mit $11,50 €$ pro Stunde sogar noch deutlich oberhalb des europäischen Spitzenwertes von Luxemburg liegt. Mit Mindestlöhnen von 7,39 bzw. 7,16€ pro Stunde liegen Neuseeland und Kanada ${ }^{3}$ etwas unterhalb der europäischen Spitzengruppe. Japan ${ }^{\boldsymbol{\oplus}}$ und die USA ${ }^{\boldsymbol{\oplus}}$ bewegen sich mit Mindestlöhnen von 6,64 $€$ und 5,21 € am oberen Rand der europäischen Mittelgruppe, während Argentinien, Kroatien und Korea mit Mindestlöhnen zwischen 2,00 und 3,00€ das untere Spektrum dieser Gruppe repräsentieren. In der Türkei hat der Mindestlohn mit 1,96€ einen mit den meisten mittel- und osteuropäischen Staaten vergleichbaren Wert erreicht. Ähnliches gilt für den gesetzlichen Mindestlohn in Brasilien, der nach kräftigen Erhöhungen in den letzten Jahren mit 1,41 € pro Stunde bereits oberhalb der Mindestlöhne in Litauen, Rumänien und Bulgarien liegt.

Die Aussagekraft eines in Euro vorgenommenen Vergleichs absoluter Mindestlohnbeträge ist jedoch begrenzt. Außerhalb der Eurozone wird dieser mitunter stark durch Wechselkursschwankungen verzerrt. Hinzu kommt, dass aufgrund des jeweiligen nationalen Preisniveaus und der damit verbundenen Lebenshaltungskosten den jeweiligen Mindestlohnbeträgen oft eine sehr unterschiedliche Kaufkraft gegenübersteht. Letzteres kann dadurch berücksichtigt werden, dass der internationale Vergleich gesetzlicher Mindestlöhne zusätzlich in Kaufkraftstandards (KKS) durchgeführt wird (Abbildung 2). Gegenüber dem nominellen Mindestlohnvergleich in Euro werden die Niveauunterschiede zwischen den nationalen Mindestlöhnen bei einem Vergleich in KKS deutlich geringer: Während die Differenz zwischen dem höchsten und dem niedrigsten Mindestlohn gemessen in Euro bei etwa 1:14 liegt, ist sie gemessen in KKS mit einem Verhältnis von 1:6 deutlich geringer. Bei der Berechnung in KKS kommt es zum Teil auch zu erheblichen Veränderungen in der Rangfolge der Staaten. So fällt z.B. Australien mit dem auf Euro-Basis höchsten Mindestlohn-
ABB. 1

\section{Gesetzliche Mindestlöhne pro Stunde 2012}

Angaben in Euro

- bis $1,99 € \quad$ - 2,00 bis $6,99 € \quad \square 7,00 €$ und mehr

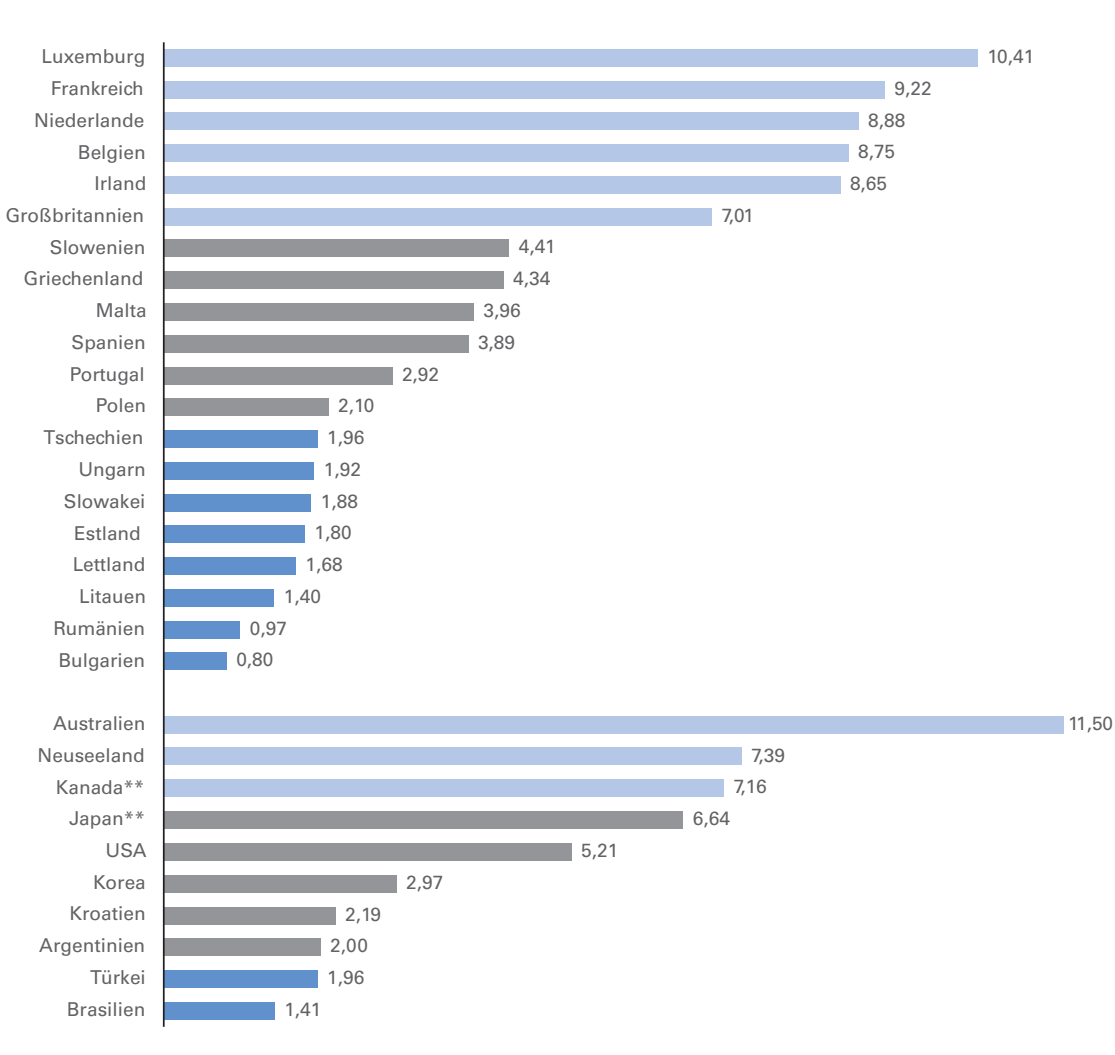

* Jeweils zum 01.01.2012; Umrechnung in Euro zum Jahresdurchschnittskurs 2011. **gewichteter Durchschnitt regionaler Mindestlöhne.

Quelle:WSI-Mindestlohndatenbank 2011.

3 In Kanada werden die Mindestlöhne auf der Ebene der Provinzen festgelegt. Da kein nationaler Mindestlohn existiert, wird hier ein nach Beschäftigtenzahl gewichteter nationaler Durchschnittswert verwendet. Der höchste und der niedrigste regionale Mindestlohn weichen hierbei um jeweils etwa $10 \%$ vom kanadischen Durchschnittsmindestlohn ab.

$(4$ Ähnlich wie in Kanada existiert auch in Japan kein einheitlicher nationaler Mindestlohn (Ohashi 2011). Gesetzliche Mindestlöhne werden vielmehr auf regionaler Ebene festgelegt. Gegenüber dem gewichteten nationalen Durchschnittswert liegen die regionalen Mindestlöhne jeweils bis zu $13 \%$ darüber oder darunter.

(5 Zusätzlich zum nationalen Mindestlohn existieren in den USA auch regionale Mindestlöhne auf der Ebene der USBundesstaaten. Anfang 2012 verfügen 17 US-Bundesstaaten und der District of Columbia über einen eigenen Mindestlohn, der über dem Niveau des nationalen Mindestlohns liegt. Der höchste regionale Mindestlohn gilt mit umgerechnet 6,70 € pro Stunde im US-Bundesstaat Washington und liegt knapp $17 \%$ über der nationalen Lohnuntergrenze. Während 2011 der nationale Mindestlohn nicht erhöht wurde, kam es in acht Bundesstaaten zu einer Mindestlohnerhöhung. Darunter waren vor allem solche Staaten, die eine Indexierung des Mindestlohns an die Preissteigerungsrate vorgesehen haben (Cooper 2011). 
ABB. 2

\section{Gesetzliche Mindestlöhne pro Stunde 2012}

Angaben in Kaufkraftstandards (KKS)*

unter 3 KKS

- 3 bis $6 \mathrm{KKS}$ mehr als $6 \mathrm{KKS}$

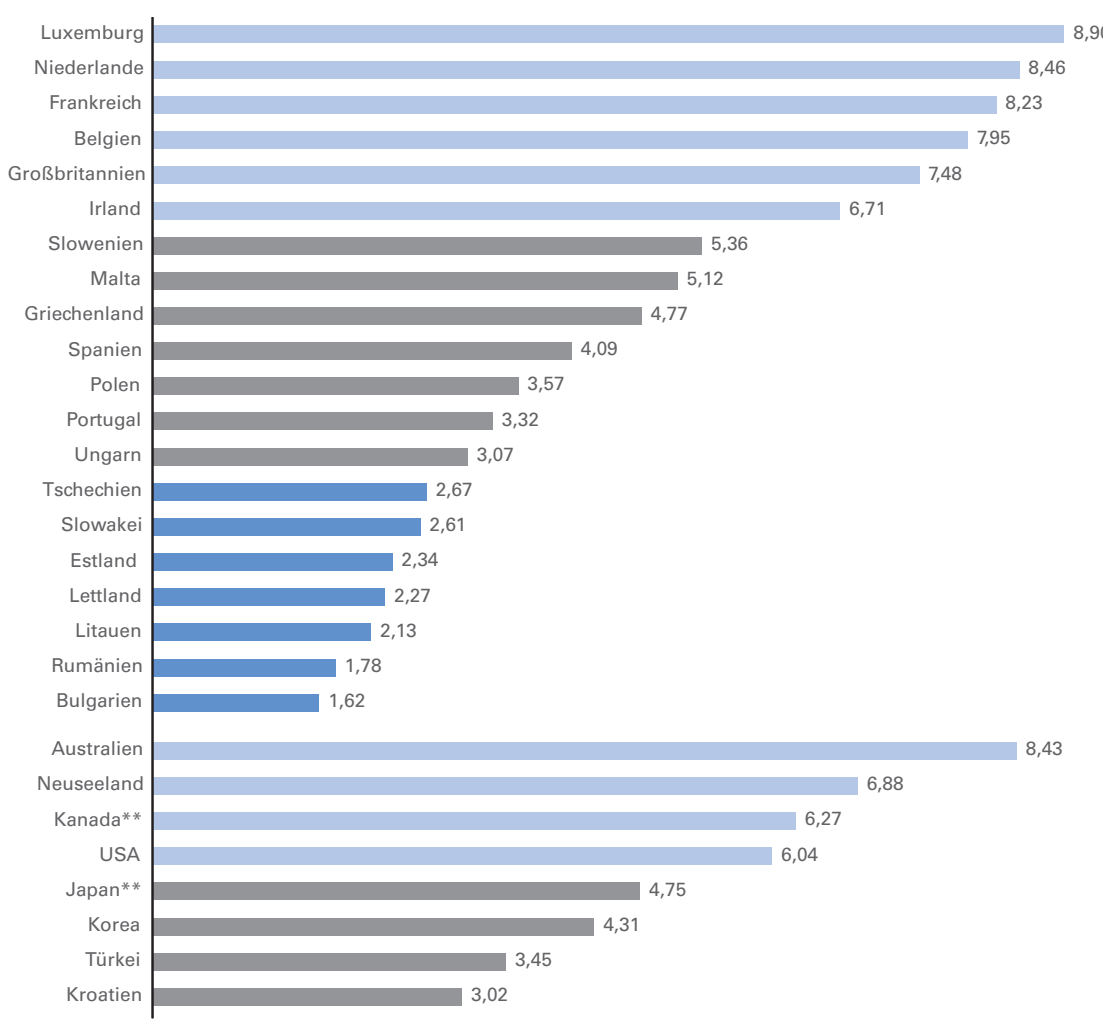

* Jeweils zum 01.01.2012, Umrechnung in KKS aufgrund der von der OECD für 2008 ausgewiesenen Kaufkraftparitäten für den privaten Konsum.

** Gewichteter Durchschnitt regionaler Mindestlöhne.

Quelle:WSI-Mindestlohndatenbank 2012.

betrag auf KKS-Basis auf den dritten Platz zurück. Luxemburg kann zwar seinen europäischen Spitzenplatz behaupten, sein Mindestlohn erfährt aufgrund der hohen Lebenshaltungskosten im Großherzogtum jedoch einen relativ starken Wertverlust. In ähnlicher Weise gilt dies auch für Frankreich, Irland und Japan, die in der Rangfolge der Länder ebenfalls Positionsverluste hinnehmen müssen. $\mathrm{Zu}$ einer relativen Positionsverbesserung kommt es hingegen bei den Niederlanden, Großbritannien und Slowenien. Den stärksten relativen Wertzuwachs erfahren auf KKS-Basis die Mindestlöhne in den mittel- und osteuropäischen Ländern sowie in der Türkei. Insgesamt bleiben jedoch auch bei einer Berechnung in KKS erhebliche Niveauunterschiede bei den Mindestlöhnen bestehen, die das tatsächliche Lohn- und Wirtschaftsgefälle zwischen den Staaten widerspiegeln.

\section{Die aktuelle Entwicklung der Mindestlöhne im Jahr 2011}

Seit Beginn des Jahres 2011 wurden insgesamt in 22 von den hier untersuchten 30 Staaten nominale Erhöhungen der Mindestlohnsätze vorgenommen (Abbildung 3). Hiervon haben allein 14 Staaten ihre Mindestlöhne zuletzt zum 1. Januar 2012 erhöht. In neun Staaten war die Anhebung der Mindestlöhne relativ moderat und bewegte sich zwischen $1,0 \%$ in Japan und 2,5 \% in Großbritannien. In weiteren acht Staaten fielen die Mindestlohnerhöhungen deutlich kräftiger aus mit Werten zwischen 3,1 \% in Malta und 8,2 \% in Polen. In fünf weiteren Staaten kam es sogar zu zweistelligen Erhöhungen der nominalen Mindestlohnsätze. Spitzenreiter war hierbei Argentinien mit einem Mindestlohnzuwachs von $25,0 \%$, gefolgt von Ungarn mit $19,2 \%$, Brasilien mit 15,1\%, Bulgarien mit 12,2 \% und der Türkei mit 11,5\%. Bei der Spitzengruppe handelt es sich allerdings ausnahmslos um Länder mit einem sehr geringen Mindestlohnniveau, sodass die starke Erhöhung auch durch entsprechende Basiseffekte beeinflusst wird.

In insgesamt acht der hier untersuchten Länder (darunter sechs EU-Staaten, dem EU-Kandidaten Kroatien und den USA) gab es 2011 keine Mindestlohnzuwächse, sodass die bestehenden nominalen Mindestlohnbeträge eingefroren wurden. Hierzu gehörten u.a. Spanien und Portugal, wo erstmals seit Jahrzehnten die bisher übliche jährliche Anpassung des nationalen Mindestlohns ausgesetzt wurde. In Kroatien und den USA wurden hingegen bereits seit 2,5 Jahren und in Litauen sogar bereits seit vier Jahren keine Mindestlohnerhöhungen mehr vorgenommen. Die mit fünf Jahren bislang längste Phase ohne Mindestlohnzuwächse erlebt die Tschechische Republik, wo die Mindestlöhne zuletzt zum 1. Januar 2007 angehoben wurden.

Eine besondere Entwicklung vollzog sich in Irland, das erstmalig in Europa vorübergehend sein nominales Mindestlohnniveau abgesenkt hat. So wurde zum 1. Februar 2011 eine Absenkung des irischen Mindestlohns um einen Euro von 8,65 auf 7,65 € pro Stunde wirksam. Nach dem Regierungswechsel in Irland wurde diese fast 12 \%ige Kürzung des Mindestlohns jedoch wieder zurückgenommen und zum 1. Juli 2011 das alte Niveau von 8,65€ pro Stunde wieder hergestellt (Slattery/Madden 2011). Insgesamt hat auch Irland nunmehr eine relativ lange Stagnationsphase bei der Entwicklung der Mindestlöhne erlebt, dessen letzte Erhöhung bis zum 1. Juli 2007 zurückreicht.

Da im Jahr 2011 die nominalen Mindestlohnsätze vielfach nur relativ schwach angehoben oder sogar eingefroren wurden, während gleichzeitig die Verbraucherpreise wieder stärker anstiegen sind, ist der inflationsbereinigte reale Wert des Mindestlohns in einer Mehrzahl von Staaten zurückgegangen (Abbildung 4). Insgesamt blieb die Anpassung der Mindestlöhne in 18 von 30 hier berücksichtigten Ländern hinter der Preissteigerungsrate zurück. Am stärksten sank der reale Mindestlohnwert mit $-4,3$ bzw. $-4,1 \%$ in Litauen 
und Lettland, gefolgt von Spanien, Portugal und den USA mit Werten zwischen $-3,2$ und $-3,7 \%$.

Von den zwölfStaaten mit einem Reallohnanstieg bei den Mindestlöhnen fiel dieser in sechs Ländern mit Zuwächsen von bis zu $2 \%$ eher moderat aus. In vier Ländern bewegte sich der Reallohnanstieg zwischen 4,0 \% in Polen und 8,5\% in Brasilien. Mit einer Steigerung von jeweils mehr als $15 \%$ kam es in Ungarn und Argentinien zu einem außergewöhnlich kräftigen Realzuwachs des gesetzlichen Mindestlohns.

Im Vergleich zum Vorjahr fiel die Entwicklung der Mindestlöhne 2011 in den meisten Ländern insgesamt deutlich schwächer aus. ${ }^{\bullet}$ Zwar lag in gut der Hälfte der hier untersuchten Staaten die nominale Mindestlohnerhöhung 2011 über der des Jahres 2010. In vielen Fällen war diese jedoch nicht in der Lage, die deutlich höheren Preissteigerungsraten im Jahr 2011 zu kompensieren. Bezogen auf den realen Wert des Mindestlohns blieb die Entwicklung in 20 von 30 Staaten hinter der des Vorjahres zurück. In elf Ländern (darunter acht EU-Staaten) war die reale Mindestlohnentwicklung bereits im zweiten Jahr in Folge rückläufig.

\section{Der relative Wert gesetzlicher Mindestlöhne (Kaitz-Index)}

Die Bedeutung des Mindestlohns wird nicht allein durch seinen absoluten Wert bestimmt, sondern auch durch seine Stellung im jeweiligen nationalen Lohngefüge. Letztere kann durch den sogenannten Kaitz-Index (benannt nach dem amerikanischen Ökonomen Hyman Kaitz) bestimmt werden, der den relativen Wert des gesetzlichen Mindestlohns zum Ausdruck bringt und statistisch als Prozentsatz des Mindestlohns vom jeweiligen nationalen Durchschnittsoder Medianlohn gemessen wird. Während der Durchschnittslohn durch das arithmetische Mittel aller Löhne bestimmt wird, ist der Medianlohn der mittlere Lohn, bei dem die Hälfte aller Beschäftigten mehr und die andere Hälfte weniger verdient. Entsprechende Datensätze mit aktuellen Werten bis 2010 werden von der OECD und von EUROSTAT veröffentlicht. ${ }^{0}$

Im Jahr 2010 zeigte der relative Wert des Mindestlohns bezogen auf den Medianlohn eine große Schwankungsbreite (Tabelle 1). Von den insgesamt 25 Ländern, für die entsprechende Daten vorliegen, bewegte sich der Mindestlohn in einer Mehrheit von 13 Staaten zwischen 40 und $50 \%$ des Medianlohns. Unterhalb dieses Niveaus befanden sich mit den USA, Japan und der Tschechischen Republik drei Staaten, deren relativer Mindestlohnwert zwischen 35 und $39 \%$ des jeweiligen Medianlohns lag. Sieben weitere Staaten (Neuseeland, Slowenien, Portugal, Australien, Lettland, Belgien und Irland) verfügten hingegen über ein deutlich höheres Mindestlohnniveau zwischen 52 und $59 \%$.

Die gemessen am Medianlohn höchsten Mindestlöhne existierten mit $60 \%$ in Frankreich und $66 \%$ in der Türkei.
ABB. 3

\section{Nominale Entwicklung gesetzlicher Mindestlöhne 2011*}

Angaben in Prozent

\begin{tabular}{|c|c|c|}
\hline Argentinien & 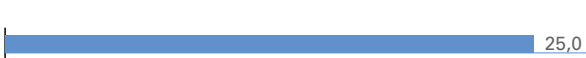 & $\begin{array}{r}\text { zuletzt erhöht: } \\
01.09 .2011 \\
\end{array}$ \\
\hline Ungarn & 19,2 & 01.01.2012 \\
\hline Brasilien & 15,1 & 01.01 .2012 \\
\hline Bulgarien & 12,2 & 01.09 .2011 \\
\hline Türkei & 11,5 & 01.01 .2012 \\
\hline Polen & 8,2 & 01.01 .2012 \\
\hline Korea & 6,0 & 01.01 .2012 \\
\hline Rumänien & 4,6 & 01.01 .2012 \\
\hline Estland & 4,0 & 01.01 .2012 \\
\hline Kanada & 3,7 & 01.01 .2012 \\
\hline Australien & 3,4 & 01.07 .2011 \\
\hline Slowakei & 3,3 & 01.01 .2012 \\
\hline Malta & 3,1 & 01.01 .2012 \\
\hline Großbritannien & 2,5 & 01.10 .2011 \\
\hline Luxemburg & 2,5 & 01.10 .2011 \\
\hline Frankreich & 2,4 & 01.01 .2012 \\
\hline Slowenien & 2,1 & 01.01 .2012 \\
\hline Belgien & 2,0 & 01.05 .2011 \\
\hline Neuseeland & 2,0 & 01.04 .2011 \\
\hline Niederlande & 1,6 & 01.01 .2012 \\
\hline Griechenland & 1,4 & 01.07 .2011 \\
\hline Japan & 1,0 & 01.01 .2012 \\
\hline Irland & 0,0 & 01.07 .2007 \\
\hline Kroatien & 0,0 & 01.06 .2009 \\
\hline Lettland & 0,0 & 01.01 .2011 \\
\hline Litauen & 0,0 & 01.01 .2008 \\
\hline Portugal & 0,0 & 01.01 .2011 \\
\hline Spanien & 0,0 & 01.01 .2011 \\
\hline Tschechien & 0,0 & 01.01 .2007 \\
\hline USA & 0,0 & 24.07 .2009 \\
\hline
\end{tabular}

*Entwicklung vom 01.01.2011 zum 01.01.2012.

Quelle:WSI-Mindestlohndatenbank 2012.

Die Ursachen für die hohen Mindestlohnwerte sind in beiden Ländern jedoch sehr unterschiedlich. Während in Frankreich das hohe relative Mindestlohnniveau durch einen vergleichsweise hohen absoluten Mindestlohnbetrag bedingt ist, erklärt sich der hohe Wert für die Türkei aus den Besonderheiten des türkischen Arbeitsmarktes. Letzterer ist durch eine hohe Einkommenspolarisierung und einen breiten informellen Sektor gekennzeichnet, in dem der gesetzliche Mindestlohn für viele Beschäftigte den Standardlohn bildet (Koçer/Visser 2009). Die Position der Türkei wird deutlich relativiert, wenn der Kaitz-Index nicht anhand des Medianlohns, sondern anhand des Durchschnittslohns berechnet wird, wo sie sich mit $35 \%$ nur noch im unteren Mittelfeld bewegt.

(6 Zu den Vorjahresdaten vgl. Schulten 2011a.

(7) Zu den methodischen Unterschieden beider Datensätze vgl Schulten 2010. Im Folgenden wird auf die Datenbank der OECD zurückgegriffen, da sie eine höhere Abdeckung der hier untersuchten Länder aufweist. 
ABB. 4

\section{Reale Entwicklung gesetzlicher Mindestlöhne 2011*}

Angaben in Prozent

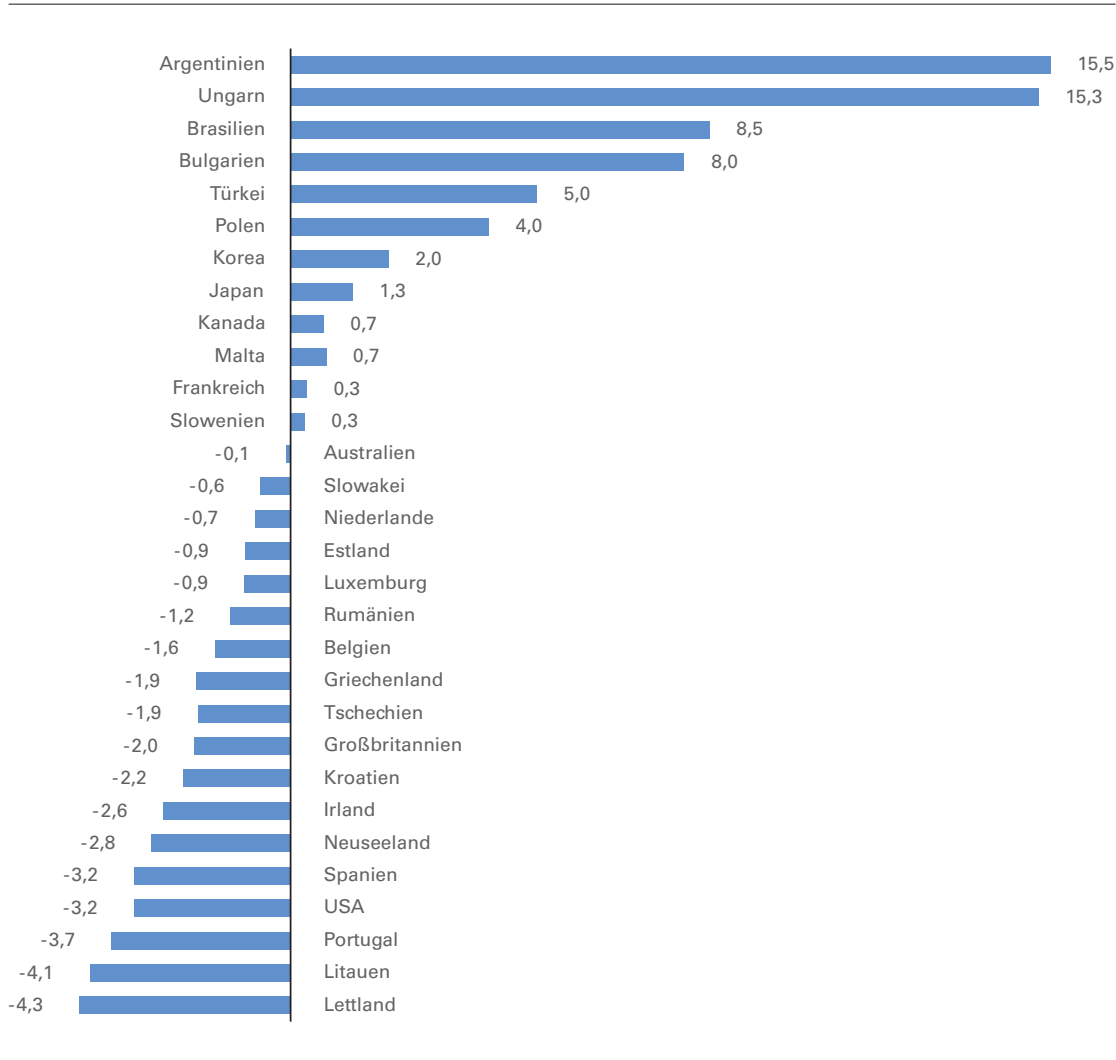

*Entwicklung vom 01.01.2011 zum 01.01.2012; nominale Erhöhung deflationiert um den nationalen Anstieg der Verbraucherpreise 2011.

In einer Mehrzahl von 15 der hier erfassten 25 Länder bewegte sich der relative Wert des Mindestlohns im Jahr 2010 zwischen 30 und $40 \%$ des Durchschnittslohns. Das Schlusslicht bildeten wiederum die USA und die Tschechische Republik, wo der Mindestlohn lediglich 28 bzw. $29 \%$ des Durchschnittslohns ausmachte. Demgegenüber verfügten sieben Länder - darunter Lettland, die Niederlande, Irland, Belgien, Australien, Slowenien und Frankreich - über einen Mindestlohn zwischen 40 und 50 \% des Durchschnittslohns. Spitzenreiter war schließlich Neuseeland, das mit einem relativen Mindestlohnniveau von $52 \%$ des Durchschnittslohns als einziges Land über der 50\%-Marke lag.

Mit der Analyse des Kaitz-Index lässt sich zeigen, dass in den meisten Staaten die gesetzlichen Mindestlöhne zum Teil deutlich - unterhalb der von OECD und EUROSTAT verwendeten Niedriglohnschwelle liegen, die bei zwei Dritteln des Medianlohns angesetzt wird. In Analogie zu der in der Armutsforschung verwendeten Armutsschwelle von $60 \%$ des Medianeinkommens oder 50 \% des Durchschnittseinkommens kann davon gesprochen werden, dass in vielen Ländern der gesetzliche Mindestlohn lediglich einen „Armutslohn“ festschreibt, der in der Regel nur ein sehr bescheidenes Einkommensniveau ermöglicht und in manchen Fällen sogar unterhalb des offiziellen Existenzminimums liegt. Aufgrund des oft sehr niedrigen Sicherungsniveaus hat sich trotz gesetzlicher Mindestlöhne in vielen europäischen Ländern ein großer Niedriglohnsektor herausgebildet (George 2011).

Das relativ niedrige Niveau vieler gesetzlicher Mindestlöhne ist in den letzten Jahren von verschiedensten Seiten kritisiert worden. Nahezu regelmäßig rügt z. B. der Europarat zahlreiche Staaten in Europa dafür, dass ihr Mindestlohnniveau nicht mit der Europäischen Sozialcharta in Einklang steht. Letztere enthält u.a. das „Recht auf ein faires Entgelt", das nach Ansicht des Europarates mindestens bei $60 \%$ des Durchschnittsentgeltes liegen sollte (Schulten 2011a). In ähnlicher Weise hat sich auch das Europäische Parlament mittlerweile in mehreren Stellungnahmen dafür ausgesprochen, dass im Rahmen einer europaweit koordinierten Mindestlohnpolitik die nationalen Mindestlöhne auf einen bestimmten europaweit zu harmonisierenden Prozentsatz des nationalen Durchschnittslohnes angehoben werden sollen. Im Jahr 2010 forderte das Europäische Parlament von der Europäischen Kommission, im Rahmen einer Studie zu überprüfen, „welche Auswirkungen eine von ihr auf den Weg gebrachte Gesetzesinitiative zur unionsweiten Festsetzung eines Mindestlohns in jedem einzelnen Mitgliedstaat hätte“ (Europäisches Parlament 2010).

Schließlich treten in vielen europäischen Ländern die Gewerkschaften und andere soziale Organisationen bereits seit Längerem für eine strukturelle Erhöhung der gesetzlichen Mindestlöhne ein, damit der Mindestlohn ein soziokulturelles Existenzminimum gewährleistet und den Anforderungen eines „Living Wage“ entspricht. In Frankreich fordert z.B. die Gewerkschaft CGT eine Erhöhung des monatlichen Mindestlohns um mehr als $20 \%$ auf $1.700 €$, was bei der in Frankreich nach wie vor als Regelarbeitszeit geltenden 35-Stunden-Woche einem Mindestlohn von 11,20€ pro Stunde entsprechen würde (CGT 2011). In Großbritannien weisen die Gewerkschaften regelmäßig darauf hin, dass der britische Mindestlohn unterhalb des Existenzminimums liegt, und plädieren für eine substanziellere Anhebung (Low Pay Commission 2011). Hinzu kommen in Großbritannien zahlreiche lokale „Living Wage-Initiativen“, die entsprechend den lokalen Lebenshaltungskosten für ein deutlich höheres lokales Mindestlohnniveau oberhalb des nationalen Mindestlohns plädieren. ${ }^{\mathbf{8}}$

8 Zu den bekanntesten Living-Wage-Initiativen gehört diejenige in London, wo die Londoner Stadtregierung bereits seit Mitte der 2000er Jahre jährlich eine Empfehlung für einen lokalen Mindestlohn verabschiedet, der vor allem über den öffentlichen Sektor und die öffentliche Auftragsvergabe umgesetzt wird. Derzeit liegt der London Living Wage bei umgerechnet 9,56 € pro Stunde und damit mehr als 18 \% höher als der nationale Mindestlohn in Großbritannien (Greater London Authority 2011). 
In einigen Ländern wie z. B. in Spanien, Portugal oder Rumänien haben sich Regierungen und Gewerkschaften in den 2000er Jahren darauf verständigt, in mehrjährigen Anpassungsschritten zu einer strukturellen Erhöhung des nationalen Mindestlohnniveaus zu gelangen. Vor dem Hintergrund der Krise sind die Anpassungspläne jedoch von den dortigen Regierungen einseitig aufgekündigt worden (Schulten 2010).

Substanziellere Mindestlohnerhöhungen, die das Ziel einer strukturellen Anhebung des Mindestlohnniveaus verfolgen, waren in den letzten Jahren eher selten. Eine Ausnahme bildete die Erhöhung des slowenischen Mindestlohns um über $30 \%$ im Jahr 2010, durch die Slowenien mittlerweile einen der höchsten relativen Mindestlohnwerte in Europa aufweist (Schulten 2011a). Außerhalb Europas haben in den letzten Jahren vor allem einige lateinamerikanische Staaten wie Argentinien und Brasilien ihre ehemals extrem niedrigen Mindestlöhne mit vielfach zweistelligen Wachstumsraten nachhaltig erhöht (ILO 2011).

\section{Fazit: Schwache Mindestlohnent- wicklung als Teil staatlicher Austeritätspolitik}

Vor dem Hintergrund einer sich wieder deutlich abkühlenden Weltkonjunktur hat sich die Entwicklung der Mindestlöhne im Jahr 2011 gegenüber dem Vorjahr noch einmal deutlich verlangsamt. Insbesondere in Europa kam es in vielen Staaten entweder nur zu geringen Mindestlohnsteigerungen oder die Mindestlöhne wurden auf dem bestehenden Niveau eingefroren. In der Mehrzahl der Länder kam es für die Mindestlohnbezieher angesichts höherer Inflationsraten zu deutlichen Reallohnverlusten. Die Mindestlöhne scheinen damit den Trend für die allgemeine Lohnentwicklung in Europa zu setzen, die in den letzten Jahren einem zunehmend restriktiven Kurs folgt (Schulten 2011b).

Die Mindestlohnpolitik wurde in vielen Ländern unmittelbar durch die vorherrschende Austeritätspolitik der Europäischen Union beeinflusst. Insbesondere in den europäischen Krisenstaaten hat die Troika aus Internationalem Währungsfond (IWF), Europäischer Kommission und Europäischer Zentralbank (EZB) mehr oder weniger direkt versucht, Einfluss auf die nationale Entwicklung der Mindestlöhne zu nehmen. So musste sich z.B. Portugal in der Vereinbarung mit dem IWF verpflichten, erst dann wieder eine Erhöhung der Mindestlöhne vorzunehmen, „wenn dies durch die ökonomischen Verhältnisse gerechtfertigt ist“ und wenn dies im Rahmen einer Überprüfung des gegenüber dem IWF von der portugiesischen Regierung zugesagten Reformprogramms vereinbart wird (IMF 2011 ). ${ }^{\circledR}$ Damit hat sich der IWF faktisch ein Veto-Recht gegen zukünftige Mindestlohnerhöhungen in Portugal zugebilligt.
TABELLE 1

\section{Gesetzliche Mindestlöhne 2010}

in Prozent der Median- und Durchschnittslöhne von Vollzeitbeschäftigten

\begin{tabular}{|c|c|c|c|}
\hline Medianlohn & & Durchschnittslohn & \\
\hline Türkei & 66 & Neuseeland & 52 \\
\hline Frankreich & 60 & & \\
\hline Neuseeland & 59 & Frankreich & 48 \\
\hline Slowenien & 58 & Slowenien & 47 \\
\hline Portugal & 56 & Australien & 45 \\
\hline Australien & 54 & Belgien & 45 \\
\hline Lettland & 54 & Irland & 44 \\
\hline Belgien & 52 & Niederlande & 42 \\
\hline Irland & 52 & Lettland & 40 \\
\hline Griechenland & 49 & Kanada & 39 \\
\hline Ungarn & 47 & Portugal & 39 \\
\hline Niederlande & 47 & Großbritannien & 38 \\
\hline Großbritannien & 46 & Litauen & 37 \\
\hline Litauen & 45 & Polen & 37 \\
\hline Polen & 45 & Slowakei & 36 \\
\hline Slowakei & 45 & Ungarn & 35 \\
\hline Kanada & 44 & Luxemburg & 35 \\
\hline Spanien & 44 & Spanien & 35 \\
\hline Rumänien & 44 & Türkei & 35 \\
\hline Luxemburg & 43 & Estland & 34 \\
\hline Estland & 41 & Griechenland & 33 \\
\hline \multirow[t]{3}{*}{ Korea } & 41 & Korea & 33 \\
\hline & & Japan & 32 \\
\hline & & Rumänien & 30 \\
\hline USA & 39 & Tschechische Republik & 29 \\
\hline Japan & 37 & USA & 28 \\
\hline Tschechische Republik & 35 & & \\
\hline
\end{tabular}

Quelle: OECD (http://stats.oecd.org/Index.aspx?DataSetCode=MIN2AVE).

Ähnlich verhält es sich in Spanien, wo unlängst bekannt wurde, dass die EZB in einem Brief an die spanische Regierung als Gegenleistung für den Kauf spanischer Anleihen die Einführung eines neuen Typs von „Mini-Job“ verlangte, der unterhalb des gesetzlichen Mindestlohns bezahlt werden sollte (El Pais vom 7. Dezember 2011). In beiden Ländern haben die Regierungen unter internationalem Druck langfristige Vereinbarungen mit den Gewerkschaften für eine strukturelle Erhöhung des Mindestlohns aufgehoben und die bestehenden Mindestlöhne eingefroren.

(9 Im englischen Original lautet die Vereinbarung: „Over the program period, any increase in the minimum wage will take place only if justified by economic conditions and agreed in the context of regular program reviews" (IMF 2011, S. 14). 
In Irland wurde Anfang 2011 zunächst ebenfalls auf Druck von IWF und EU der bestehende Mindestlohn um einen Euro gekürzt. Nachdem die irische Opposition u.a. mit der Forderung nach Rücknahme dieser Kürzung die Wahlen gewonnen hatte, stimmte schließlich auch die Troika einer Rücknahme zu, jedoch nur unter der Bedingung, dass hierfür die Unternehmen bei der Zahlung von Sozialversicherungsbeiträgen entlastet würden (Slattery/Madden 2011). In Griechenland grenzte es angesichts des massiven Drucks der Troika fast schon an ein Wunder, dass sich Arbeitgeber und Gewerkschaften Mitte 2011 auf eine zumindest leichte Erhöhung des nationalen Mindestlohns verständigten. Mittlerweile hat die griechische Regierung jedoch den Forderungen der Troika nachgegeben und gegen den gemeinsamen Widerstand von Arbeitgebern und Gewerkschaften beschlossen, den Mindestlohn um 22 \% abzusenken (Frankfurter Rundschau vom 9. Februar 2012).

In den westeuropäischen Ländern wie Frankreich oder den BeneluxStaaten wurde die Anpassung der Mindestlöhne auf das gesetzlich vorgegebene Mindestmaß begrenzt und beschränkte sich im Wesentlichen auf den Ausgleich der Inflationsrate. In Großbritannien blieb die Erhöhung des Mindestlohns hingegen bereits im zweiten Jahr in Folge deutlich hinter der Preissteigerungsrate zurück. Als eines der wenigen Länder in Europa hat sich ausgerechnet das rechtspopulistisch geführte Ungarn gegen den allgemeinen Austeritätsdruck der EU gerichtet. Die ungarische Regierung setzte einseitig eine starke Erhöhung des Mindestlohns durch, nachdem sie zuvor die Kooperation mit den Gewerkschaften in der Mindestlohnpolitik aufgekündigt hatte (Komiljovics 2011).

Obwohl zwischen der aktuellen Verschuldungsproblematik und der Entwicklung der Mindestlöhne kein direkter ökonomischer Zusammenhang besteht, so wird unter dem Druck der EU eine restriktive Mindestlohnpolitik mittlerweile als fester Bestandteil der derzeit vorherrschenden Austeritätspolitik gesehen. Legitimiert wird diese Politik durch den Hinweis, dass die Mindestlöhne ein wichtiger Indikator für die allgemeine Lohnentwicklung sind und als solche unmittelbar Einfluss auf die Wettbewerbsfähigkeit haben, deren Verbesserung wiederum als Königsweg zur Überwindung der Schuldenkrise gilt. ${ }^{\oplus}$ Mit der vorherrschenden Kombination aus rigider Sparpolitik und restriktiver (Mindest-)Lohnpolitik werden die Potenziale zur Entwicklung der Binnenwirtschaft jedoch systematisch abgewürgt. Die Hoffnung, durch eine europäische Verallgemeinerung des Exportmodells Deutschland aus der Krise zu kommen, dürfte sich hingegen ebenfalls als trügerisch erweisen. Damit wird jedoch das von kritischen Ökonomen bereits seit Längerem befürchtete Szenario einer sich verfestigenden Stagnationsperiode mit einer stark deflationären Grundtendenz immer wahrscheinlicher. Umso wichtiger ist es, dass zukünftig von der Lohnpolitik wieder deutlich dynamischere Impulse ausgehen. Gerade weil der Mindestlohn in vielen Ländern eine wichtige Signalwirkung für die allgemeine Lohnentwicklung hat, ist seine zukünftige Entwicklung hierbei von entscheidender Bedeutung (Herr/Kazandziska 2011).

\section{LITERATUR}

Confédération Générale du Travail (CGT) (2011): SMIC - Intervention de la délégation CGT à la Commission Nationale de la Négociation Collective, 20. Dezember, http://www.cgt.fr/spip.php?page=article_dossier1\&id_article=38868 Cooper, D. (2011): More than a million workers helped by inflation-linked state minimum wage increases, Economic Policy Institute, 20. Dezember

Europäisches Parlament (2010): Bedeutung des Mindesteinkommens für die Bekämpfung der Armut und die Förderung einer integrativen Gesellschaft in Europa, Entschließung vom 20. Oktober, INI/2010/2039

George, R. (2011): Niedriglohn und Geschlecht im europäischen Vergleich, in: WSI-Mitteilungen 64 (19), S. 548-556, http://www.boeckler.de/wsimit_2011_10_ george.pdf

Greater London Authority (2011): A Fairer London: The 2011 Living Wage in London http://www.london.gov.uk/sites/default/files/living-wage-2011.pdf Groupe d'experts sur le SMIC (2011): Salaire Minimum Interprofessionnel de Croissance, Bericht der Expertengruppe an die nationale Tarifkommission, Dezember, http://static.lentreprise.com/pub/pdf/Le-rapport-du-groupe-d-expertssur-le-SMIC-decembre-2011.pdf

Herr, H./Kazandziska M. (2011): Principles of Minimum Wage Policy - Econom ics, Institutions and Recommendations, Global Labour University Working Paper (11), Berlin

International Labour Organisation (ILO) (2010): Wages policies in time of crisis, Global Wage Report 2010/11, Genf

International Labour Organisation (ILO) (2011): Panorama Laboral 2011. América Latina y el Caribe, Lima

International Monetary Fund (IMF) (2011): Portugal: Letter of Intent, Memorandum of Economic and Financial Policies and Technical Memorandum of Understanding, 20. Mai, http://www.imf.org/external/np/loi/2011/prt/051711.pdf Koçer, R.G./Visser, J. (2009): The Role of the State in Balancing the Minimum Wage in Turkey and the USA, in: British Journal of Industrial Relations 47 (2), S. $349-370$

Komiljovics, M. (2011): Hungary: Government to stop consulting unions on minimum wage, EIROnline, http://www.eurofound.europa.eu/eiro/2011/07/ articles/hu1107021i.htm

Low Pay Commission (2011): National Minimum Wage Report 2011, April, http://www.lowpay.gov.uk/lowpay/report/pdf/Revised_Report_PDF_with_April_ date.PDF

Ohashi, I. (2011): The Minimum Wage System in Japan: In Light of Circumstances in the United States and Europe, in: Japan Labour Review 8 (2), S. $4-23$

Schulten, T. (2009): WSI-Mindestlohnbericht 2009, in: WSI-Mitteilungen 62 (3), S. 150-157, http://www.boeckler.de/wsimit_2009_03_schulten.pdf

Schulten, T. (2010): WSI-Mindestlohnbericht 2010 - Unterschiedliche Strategien in der Krise, in: WSI-Mitteilungen 63 (3), S. 152-160, http://www.boeckler.de/ wsimit_2010_03_schulten.pdf

Schulten, T. (2011a): WSI-Mindestlohnbericht 2011 - Mindestlöhne unter Krisendruck, in: WSI-Mitteilungen 64 (3), S. 131-137, http://www.boeckler.de/ wsimit_2011_03_Schulten.pdf

Schulten, T. (2011 b): Europäischer Tarifbericht des WSI 2010/2011, in: WSI-Mitteilungen 64 (7), S. 355-362, http://www.boeckler.de/wsimit_2011_07_schulten. pdf

Slattery, L./Madden C. (2011): Minimum wage restored to $€ 8.65$ an hour under terms of revised deal, in: The Irish Times vom 16. April

\section{AUTOR}

THORSTEN SCHULTEN, Dr., Wissenschaftler im Wirtschafts- und Sozialwissenschaftlichen Institut (WSI) der Hans-Böckler-Stiftung. Arbeitsschwerpunkte: Arbeits- und Tarifpolitik in Europa.

thorsten-schulten@boeckler.de
(10 So argumentiert z.B. die französische Wissenschaftskommission, die jährreich erstellt und auf dieser Grundlage eine Empfehlung für die Erhöhung des französischen Mindestlohns ausgibt (Groupe d'experts sur le SMIC 2011). 